Bericht über den SAMW-Workshop «Zertifizierung im medizinischen Kontext: Chancen und Risiken» vom 26.4.2013 in Bern

\title{
«Zertifizitis» oder Wer zertifiziert die Zertifizierer?
}

\author{
Sibylle Ackermann Birbauma, \\ Hermann Amstad ${ }^{b}$, \\ Urs Metzgerc \\ a lic. theol., dipl. biol., \\ wiss. Mitarbeiterin SAMW \\ b Dr. med., Generalsekretär \\ SAMW \\ c Prof. Dr. med., \\ ehem. Chefarzt Chirurgie \\ Stadtspital Triemli, \\ Präsident der Begleitkommis- \\ sion Qualitätsempfehlungen, \\ Uitikon
}

Korrespondenz:

Schweizerische Akademie der Medizinischen Wissenschaften Petersplatz 13

CH-4051 Basel

mail[at]samw.ch
Das Gesundheitssystem ist in Diagnostik und Therapie einem steten und raschen technologischen Wandel unterworfen. Die Vermehrung des medizinischen Wissens sowie die immer komplexeren und in rascherer Abfolge angebotenen Abklärungs- und Behandlungsoptionen erschweren zunehmend die Übersicht über Umfang und Nutzen der vorhandenen Angebote. Ärzte, Spitäler und weitere Leistungserbringer im Gesundheitswesen sind gesetzlich verpflichtet, sich über die Qualität ihrer Leistungen auszuweisen. Als Reaktion darauf gibt es eine zunehmende Zahl von Zertifikaten bzw. Qualitätslabels. Häufig ist jedoch unklar, wofür diese Zertifikate stehen, auf welchen Grundlagen sie beruhen und welches ihre Gültigkeitsdauer ist. Diese Situation hat die FMH bewogen, 2011 durch die Schweizerische Akademie der Medizinischen Wissenschaften (SAMW) Empfehlungen zur «Zertifizierung im medizinischen Kontext» ausarbeiten zu lassen (www.samw.ch/de/Publikationen/ Empfehlungen.html). Diese richten sich an Zertifizierungsstellen, Leistungserbringer, Versicherungen und Behörden.

Es gibt Hinweise darauf, dass Zertifikate immer mehr an Bedeutung gewinnen. So drängt z. B. die Krebsliga darauf, dass Frauen mit Brustkrebs nur noch an zertifizierten «Brustzentren» behandelt werden. Der Leistungsauftrag des Kantons Zürich für Palliative-Care-Institutionen hält fest, dass nach 2014 eine Zertifizierung zwingend ist. Auch das Beschlussorgan «Hochspezialisierte Medizin» verlangt in gewissen Bereichen (z. B. Stroke) Zertifizierungen, damit ein Spital diese Behandlungen noch anbieten kann.

Zertifikate werden meist auf der Basis von Qualitätskriterien vergeben. Im Prozess der Erarbeitung dieser Kriterien verständigen sich Fachvertreter(innen) auf die wesentlichen Voraussetzungen für gute Qualität medizinischer Diagnostik und Behandlung. Führt die Vergabe von Zertifikaten aber tatsächlich zu einer Verbesserung der Qualität medizinischer Leistungen? Es gibt viele offene Fragen:

- Wer gibt den Auftrag zu einer Zertifizierung?

- Wie wird die Unparteilichkeit des Zertifizierungsprozesses gewährleistet?

- Wie steht es um die interprofessionelle Erarbeitung der normativen Grundlagen?

- Welches sind die Schnittstellen zwischen Politik, Fachgesellschaften und Zertifizierungsstellen?
- Wie sehen die tarifarischen Konsequenzen von Zertifizierungen aus?

- Wer finanziert die Zertifizierungen?

Diese Situation hat die SAMW veranlasst, am 26. April 2013 in Bern zu einem Experten-Workshop einzuladen. Der Workshop sollte Gelegenheit bieten, auf diese und ähnliche Fragen einzugehen, indem er den aktuellen Stand und die Perspektiven im Bereich Zertifizierung darstellte und an einem aktuellen Beispiel die Hintergründe, den Prozess, die Umsetzung sowie die Folgen aufzeigte. Gleichzeitig sollte geklärt werden, ob für die SAMW nach der Veröffentlichung der Empfehlungen «Zertifizierung im medizinischen Kontext» weiterer Handlungsbedarf besteht. Rund 80 Expertinnen und Experten aus der ganzen Schweiz folgten der Einladung zu diesem Workshop.

\section{Stärken und Schwächen der Zertifizierung}

Prof. Dieter Conen, Präsident der Stiftung für Patientensicherheit, verglich im ersten Referat die zunehmende Zahl von Zertifikaten und Labels mit einem undurchdringlichen Urwald: Legitimation, Bedeutung, Gültigkeitsdauer und Qualitätsrelevanz von Zertifikaten seien sehr oft nicht zu erkennen. Triona Fortune, stv. CEO der International Society for Quality in Health Care, betonte im zweiten Referat die Schwierigkeit, den Nutzen von Zertifizierungsprozessen zu messen. Die Ausführungen der beiden Fachleute zeigten, dass ein Mehrwert von Zertifikaten nicht immer gegeben ist. Die bislang publizierten wissenschaftlichen Studien zeichnen ein widersprüchliches Bild darüber, ob Zertifizierung effektiv wirksam ist. Untersuchungen haben gezeigt, dass sich im Hinblick auf Patientenzufriedenheit und Behandlungssicherheit zertifizierte Spitäler nicht von nichtzertifizierten unterscheiden. Andere Studien hingegen belegen, dass Zertifizierung einen positiven Einfluss auf Strukturen und Prozesse hat. Anders als in der Industrie ist im Gesundheitssystem die Prozessqualität eher sekundär; viel entscheidender ist die Ergebnisqualität, gemessen an verschiedenen Outcome-Indikatoren. Hier sind die Forschungsresultate allerdings (noch) zu wenig eindeutig. Es besteht das Risiko, dass sich trotz beträchtlichem Aufwand für Einführung und Aufrechterhaltung einer Zertifizierung die Ergebnisqualität nicht verbessert. Das nicht 
zu vernachlässigende Ausmass an finanziellen und personellen Ressourcen, welche die Zertifizierung und Re-Zertifizierung binden, wurde immer wieder als Risiko genannt. Eine weitere Gefahr liegt darin, dass der Erwerb eines Qualitätslabels vorwiegend dem Marketing dient.

Neben den Defiziten wurde auch eine ganze Reihe von Chancen erörtert. Prof. Jean-Blaise Wasserfallen von der Direktion des CHUV in Lausanne betonte die Vorteile einer klaren und transparenten Definition von Standardabläufen und Rollenverteilungen. Übereinstimmend hielten die Fachleute fest, dass Zertifizierungsprozesse die Auseinandersetzung mit dem Thema «Qualität in der Medizin» fördern und Veränderungen auslösen; je nach Bereich kommt es zu eigentlichen Professionalisierungsschüben. Letzteres ist etwa im ambulanten Bereich (z. B. Spitex) zu beobachten, wo aber die Zertifizierung insgesamt noch weniger weit entwickelt ist. Die Mitarbeiterführung, der Ablauf von Prozessen und die Kommunikation werden verbessert, die Selbstreflexion der Beteiligten steigt, und es entsteht eine «lernende Organisation», die durch die regelmässigen Re-Zertifizierungen immer wieder mit dem Blick von aussen konfrontiert wird, der Verbesserungspotential aufdeckt. Nicht zu unterschätzen ist auch die Tatsache, dass die Auszeichnung von Personen und Einheiten für ihre gute Arbeit die Mitarbeitenden motiviert, weiterhin gute bzw. noch bessere Qualität zu leisten.

\section{Führt die Vergabe von Zertifikaten aber tatsächlich zu einer Verbesserung der Qualität medizinischer Leistungen?}

\section{Qualitätsansprüche zwischen «gut genug» und Exzellenz}

Um normative Grundlagen für das Beurteilungsverfahren zu erstellen, muss definiert werden, was gute Qualität ist. Am Beispiel der laufenden Zertifizierung von Hirnschlagzentren in der Schweiz (Stroke Unit/ Center) konkretisierte Prof. Marcel Arnold vom Inselspital Bern das Vorgehen: Für die Zertifizierung müssen verschiedene Kriterien - etwa jederzeitige Verfügbarkeit spezialisierter Fachärzte oder Anforderungen an die Infrastruktur für Diagnose, Behandlung und Monitoring der Patienten - erfüllt sein, dies im Sinne von Minimalanforderungen («minimal requirements») an Strukturen und Prozessen. Man geht davon aus, dass damit insgesamt auch die Ergebnisqualität verbessert wird. Diesbezügliche konkrete Messungen sind aber (noch) nicht vorgesehen.

Angeregt durch dieses Beispiel und durch sechs Kurz-Inputs, welche die Sicht von Ärzteschaft, Spitälern, Versicherern, Zertifizierern, Behörden und Patienten einbrachten, wurde der Qualitätsbegriff ein- gehend diskutiert. Statt an Minimalstandards könnte sich die Qualitätsprüfung auch am bestmöglichen Standard (Exzellenz) orientieren. Und welches Gewicht soll einzelnen Aspekten guter Qualität zukommen - geht es vorwiegend um Sicherheit? oder Effizienz? oder Wirksamkeit? oder um die Zugänglichkeit?

Einig waren sich alle Referenten und Diskussionsteilnehmer, dass die Definition der Qualitätskriterien aufgrund wissenschaftlicher Standards erfolgen und auf einem breiten fachlichen Konsens beruhen soll. $\mathrm{Zu}$ Diskussionen Anlass gab die Frage, wer bei der Festlegung der Kriterien einbezogen werden soll. Wissenschaftliche Studien haben beispielsweise gezeigt, dass die Patientenzufriedenheit nicht mit dem medizinischen Outcome korreliert ist. Deshalb wurde betont, dass der Qualitätsbegriff nicht einseitig definiert werden dürfe. Eine alleinige Orientierung an der (Patienten-)Sicherheit reiche nicht, auch Aspekte wie die Betreuungsqualität sollen einfliessen. Es wurde ausdrücklich vor der «Industrialisierung» der Medizin gewarnt.

\section{Weiterhin Klärungsbedarf}

Die Diskussionen verliefen sehr intensiv, und trotz der gutstrukturierenden Moderation von PD Dr. Christoph Cottier, Geschäftsführer von SanaCERT, konnten viele Aspekte nur andiskutiert werden. Klar geworden ist, dass die Bedeutung von Zertifikaten nicht überschätzt werden darf. Weder generieren sie per se Qualität, noch garantieren sie automatisch eine gute Qualität der medizinischen Versorgung. Damit Zertifizierungsprozesse wirksam sind, braucht es eine umfassende prospektive Datenerhebung mit validierten Qualitätsindikatoren (Outcome-Forschung) und deren Vergleich mit andern Institutionen (Benchmark). Gefordert wurde auch, Zertifizierungsprozesse transparenter und besser verständlich zu machen. Zudem darf Zertifizierung nicht isoliert betrachtet werden, sondern als ein Instrument zur Qualitätssicherung und -verbesserung - gleich wie motivierende Anreize für einen verbesserten Umgang mit Fehlern oder Trainings zur Förderung der Medikamentensicherheit.

Qualitätsindikatoren und Ergebnismessungen sind die Grundlagen für den Qualitätsnachweis. Die SAMW-Empfehlungen für die «Zertifizierung im medizinischen Kontext» fordern denn auch, dass die verwendeten Indikatoren die Beurteilung wesentlicher diagnostischer und therapeutischer Prozesse und die Beurteilung der Behandlungsergebnisse auch im nationalen oder internationalen Vergleich ermöglichen müssen. Nur wenn dies gelingt, macht eine Zertifizierung überhaupt Sinn und dient dem Patienten.

Alle Präsentationen des Workshops sind unter www. samw.ch/de/Projekte/Qualitaet-Medizin.html abrufbar. 